\title{
A Sensitivity Analysis of Short-Term Hydropower Planning Using Stochastic Programming
}

\author{
Yelena Vardanyan, Mikael Amelin \\ Electric Power Systems \\ Royal Institute of Technology \\ Teknikringen 33, 10044 Stockholm, Sweden \\ Email: yelena.vardanyan@ee.kth.se \\ Email: amelin.kth.se
}

\begin{abstract}
Huge amount of uncertainties are being introduced to the power market because of the ongoing growth in the renewable energy sources like wind and solar power. The intermittent nature of these power sources increases the volatility of the day-ahead market prices. Therefore, improving planning tools and constructing an optimal bidding strategy to the day-ahead market is an essential task for the price-taker hydropower producer. This paper applies an optimal bidding model under the uncertainties of the day-ahead market prices and the water inflow level. Specifically, the model is built using a two-stage stochastic mixed integer linear programming approach. The uncertainties are handled by generating scenarios based on historical data. The model is tested by studying three reservoir test system. Profound sensitivity analysis is provided, in terms of volatility in day-ahead market prices and water inflow level as well as in terms of water opportunity cost and initial volume of the reservoir. Based on the comparison of the stochastic and corresponding deterministic problems, the result aims to show the impact of modeling the uncertainties explicitly.
\end{abstract}

Keywords-Stochastic programming, optimal bidding, dayahead market, scenario generation

\section{List of Algebraic Figures}

A. Sets

i index for possible bid prices $\mathrm{i}=1, \ldots, \mathrm{I}$;

$\mathrm{j} \quad$ index for power plants $\mathrm{j}=1, \ldots, \mathrm{J}$;

$\mathrm{n}$ index for discharging segments $\mathrm{n}=1, \ldots, \mathrm{N}$;

$\mathrm{k} \quad$ index inflow scenarios $\mathrm{k}=1, \ldots, \mathrm{K}$;

$\mathrm{s} \quad$ index spot market price scenarios $\mathrm{s}=1, \ldots, \mathrm{S}$;

$\mathrm{t} \quad$ index for planning periods $(\mathrm{h}) \mathrm{t}=1, \ldots, \mathrm{T}$;

$R_{j} \quad$ the set of indices for power plants downstream hydropower plant $\mathrm{j}$;
B. Parameters

$P^{s} \quad$ probabilities for spot market price scenarios;

$\pi^{k} \quad$ probabilities for inflow scenarios;

$I_{j, t}^{k} \quad$ inflow level to each power plant at each scenario and time;

$\bar{G}_{j} \quad$ maximum power production at plant j;

$\bar{m}_{j} \quad$ maximum reservoir content;

$m_{j}^{o} \quad$ initial reservoir content;

$m_{j}^{T} \quad$ reservoir content at the end of the planning period;

$\mu_{j, n} \quad$ marginal production equivalent at plant $\mathrm{j}$ segment $\mathrm{n}$;

$\gamma_{j} \quad$ expected future production equivalent for plant $\mathrm{j}$;

$\bar{Q}_{j} \quad$ maximum discharge level at plant $\mathrm{j}$;

$q_{\bar{j}, n} \quad$ maximum discharge level in plant $\mathrm{j}$ at segment $\mathrm{n}$;

$\lambda_{f} \quad$ expected future electricity price;

$\lambda_{t}^{s} \quad$ realized spot market price for each hour and scenarios;

$\rho_{i} \quad$ possible fixed bid prices;

$\tau_{j} \quad$ the delay time for the water between power plants;

C. Variables

$G_{j, t}^{s, k} \quad$ generation level at each power plant, hour and scenarios;

$m_{j, t}^{s, k} \quad$ content of reservoir $\mathrm{j}$ at the end of hour $\mathrm{t}$, for each scenarios;

$Q_{j, t, n}^{s, k} \quad$ discharged volume for hourly bids, for each power plant, segment, hour and scenarios;

$S_{j, t}^{s, k} \quad$ spillage from reservoir $\mathrm{j}$ during hour $\mathrm{t}$, for each scenarios;

$w_{i, t} \quad$ hourly bid volumes corresponding to the possible bid prices;

$v_{t}^{s, k} \quad$ dispatch level for each hour, price and inflow scenarios;

$z \quad$ objective function value;

\section{INTRODUCTION}

Huge increase is expected in wind power introduction to the power system by 2030 [1]. This has to be well integrated into the grid changing the prevailing combination of power plants. 
The wind power production variations are huge, because the wind power forecast quality is low comparatively. These variations must be balanced by conventional units. Due to the fluctuations in the wind power, the need for balancing power will increase, which in its turn will increase the real-time market prices. Because its flexibility and capability to increase production very fast, hydropower with reservoirs can provide balancing power and earn extra profit. Therefore, there is a value of improved planning tools, which allow the hydropower producer to provide even more flexibility.

Within the past twenty years significant efforts were made throughout the world to deregulate electricity markets. In the deregulated settings the electric power is traded in a competitive way and producers are forced to have more market oriented approaches. The power trading can take place on the power exchange day-ahead market, or on the real-time market.

On one hand the increased amount of wind power in the electricity system, on the other hand the restructured nature of power sector made the short-term planning and the operation of the hydropower more and more sophisticated. This phenomenon caused the increased interest in short-term hydropower optimization problems under uncertainty.

One advantage with the hydropower is the possibility to store energy, which allows the hydropower producer to act according to the market prices. This means, the hydropower producer has an opportunity to dispatch and sell power when the prices are high and store water for the future usage while the prices are not so attractive. Therefore the optimal bidding strategy is extremely relevant to hydropower producers.

Short-term hydropower planning addresses to the hour by hour physical operation of hydropower, which includes: the spot market bidding, the generation planning based on the dayahead market dispatch, and real time balancing. The short-term hydropower planning and bidding process under uncertainty can be modeled as a multi-stage stochastic program. For those problems random variables are described by distributions. Only trivial problems are possible to solve with continuous distributions. Therefore, in most of the cases, the continuous distribution of the random variable is approximated by a discrete distribution. This discretization is known as a scenario tree or an event tree.

The main purpose of the short-term hydropower planning is to maximize the obtained profit from the hydropower generation; to find the balance between the current and future profit. There are many stochastic models for the short-term hydropower operation and bidding strategy in the literature. [2] introduces two stage stochastic model for bidding strategies of a hydropower producer under uncertainty of the day-ahead market prices. However, in this paper the uncertainty related to inflow is also considered. [3] develops multi-stage stochastic model under uncertainty of the spot market prices and water inflow, where the first stage considers one-day production plan, while the other stages relates to the production of the following six days. As in [3], this paper also considers uncertainty related to the spot market prices and water inflow, however the stochastic model in this paper aims to address bidding strategy to the spot market for the price taker hydropower producer. Bidding problem faced by a thermal price-taker producer under price uncertainty is addressed in [4]. The bidding strategy is presented using the prediction of the market prices. Then, using the expected prices, the expected-value problem is solved. Finally, based on the optimal solution bidding curves are build. In contrast, this paper generates spot market price and inflow scenarios to address the uncertainties.

Further stochastic hydropower planning models are cited in the [5]-[7]. A stochastic hydropower planning model, which takes into account uncertainty related to the real-time market prices is provided in [5]. A hydro-electric unit commitment model subject to uncertain demand is developed in [6]. A twostage stochastic optimization problem to study joint optimization of a wind farm and a pumped-storage unit in a market environment is presented [7].

This paper aims to provide profound sensitivity analysis of a short-term hydropower planning for a price-taker hydropower producer in a pool-based electricity market, under uncertainty related to the day-ahead market prices and water inflow to the reservoirs. A price-taker hydropower producer is small and don't have an influence on the market prices. Moreover, it is assumed that there is no correlation between spot market price scenarios and inflow scenarios.

The structure of the present paper is the following: section III represents planning model: background and the mathematical formulation, section IV describes method for time series analysis and develops forecasting model for the spot market prices, section $\mathrm{V}$ presents a case study based on three Swedish hydropower plants. Section VI concludes the paper.

\section{PlanNing MODEL}

\section{A. Day-ahead market bidding}

Electricity market players can exchange power on hourly bases for each hour of the following day on the day-ahead market. Selling and purchasing bids for a specific hour should be submitted previous day before the spot market is closed (the exact closing time is different in different markets, e.g. in Nordic market it is before noon). Then, according to the price, selling bids are listed in ascending order and purchasing bids in descending order. The cross point of two curves decides the market price and the volume to be dispatched for each hour. Then, all electricity players are informed about the price and volume to be traded for each hour in coming day.

The price-taker hydropower producer has to develop a bidding strategy to the day-ahead market and plan the next day generation according to the day-ahead market commitment. In order to model the bidding process, the possible bidding prices are fixed: equidistance price points are selected and the corresponding bid volumes are considered as variables (similar to the approach in [2]). Let $i$ be the index for the possible bid prices and $\rho_{i}$ represents these prices. Then, a fundamental rule is applied to couple bid volumes $w_{i, t}$ and dispatched volumes $v_{t}$ : for each hour if $\rho_{i} \leq \lambda_{t} \leq \rho_{i+1}$ then $v_{t}=\sum_{p=0}^{i} w_{i-p, t}$, where $p=1,2 \ldots i$. However, there is a data 
uncertainty related to the day-ahead market prices and water inflow. Since the day-ahead market prices for each hour are known after the market is cleared, the hydropower producer is bidding under uncertainty of day-ahead market prices. In addition, the dispatched volume to the spot market will depend on the inflow level as well. Hence, the coupling rule will have the following form: if $p_{i} \leq \lambda_{t}^{s} \leq p_{i+1}$ then $v_{t}^{s, k}=\sum_{p=0}^{i} w_{i-p, t}$.

To deal with the uncertainty multi-stage stochastic programming framework is suggested in the literature [8], [9]. Stochastic programming addresses optimization under uncertainty, and reflects the fact that new information about the uncertain data arrives as time evolves along the planning horizon. Here the bids are submitted under uncertainty of cleared market prices, hence $w_{i, t}$ variables can be considered as the first stage decision variables and have to be decided before the uncertain future is known. However, the second stage decisions, called recourse decisions, are taken after some of the unknown parameters are known. In this model the second stage involves the production aspects based on the realization of the future market prices and inflow. Therefore, the secondstage variables are indexed by the $s \in S$ and $k \in K$ scenarios superscripts: $v_{t}^{s, k}, Q_{j, t, n}^{s, k}, G_{j, t}^{s, k}, m_{j, t}^{s, k}, S_{j, t}^{s, k}$ and $u_{j, t}^{s, k}$.

\section{B. Mathematical formulation}

The mathematical formulation of the two-stage stochastic optimization problem applicable for power plants located in a row is presented below.

$$
\operatorname{Max} \sum_{s, k} P^{s} \pi^{k}\left[\sum_{t}\left(\lambda_{t}^{s} v_{t}^{s, k}+\lambda_{f} \sum_{j} \sum_{r \in R_{j}} \gamma_{r} m_{j}^{T}\right]\right.
$$

s.t.

$$
\begin{gathered}
v_{t}^{s, k}=\sum_{p=0}^{i} w_{i-p, t} \quad \text { if } \quad p_{i} \leq \lambda_{t}^{s} \leq p_{i+1}, \\
G_{j, t}^{s, k} \leq \sum_{n} \mu_{j, n} Q_{j, t, n}^{s, k}, \\
Q_{j, t, n}^{s, k} \leq \bar{q}_{j, n}, \\
m_{j, t}^{s, k} \leq \bar{m}_{j}, \\
m_{j, t}^{s, k}=m_{j, t-1}^{s, k}-\sum_{n} Q_{j, t, n}^{s, k}-S_{j, t}^{s, k}+\sum_{n} Q_{j-1, t-\tau_{j}, n}^{s, k} \\
+S_{j-1, t-\tau_{j}}^{s, k}+I_{j, t}^{k}, s \in S, t \in T, k \in K, \\
v_{t}^{s, k}=\sum_{i} G_{j, t}^{s, k}, s \in S, t \in T, k \in K,
\end{gathered}
$$

all variables $\in R_{+}$.

This is a maximization problem, since any producer intends to maximize its profit. In the model water delay time between reservoirs are considered. Besides, the water opportunity cost is estimated based on the analysis of the financial markets. (1) is the objective function, which maximizes the total profit. The first part of the objective function describes the profit from selling the water in the day-ahead market. Whereas the second part considers the profit from the stored water. Constraint (2) couples the first and second stage variables; expresses the correlation between the bid volumes and volumes dispatched. Constraints (3) bounds hourly generation for each operated power plant according to its maximum generation capacity and marginal production equivalent. It is assumed that the marginal production equivalents have decreasing manner: $\mu_{j, n}>\mu_{j, n+1}$, otherwise additional bianary variables are necessary. [10] Constraint (4) states bound for hourly discharge level. Similarly (5) puts bound for the hourly water storage level. Constraint (6) keeps the hydrological balance between reservoirs: for each hour the reservoirs new contents are equal to its old contents plus water inflow and minus water outflow. The discharge and the spillage from the upper reservoir flow in the reservoir located downstream with some delay time. Note that the delay time for the first power plant $\tau_{1}=0$. Constraint (7) is the generation balance constraint, according to which hourly power generation follows the day-ahead market dispatch.

\section{TIME SERIES ANALYSIS ARIMA}

To solve the optimization problem formulated in the previous section, hourly day-ahead market prices and water inflow levels must be predicted accurately. One of the commonly used forecasting techniques to analyze day-ahead market price time series is Autoregressive Integrated Moving Average (ARIMA) [11]. A specific class of stochastic processes which incorporates a wide range of non-stationary series, is provided by the ARIMA processes, which after differencing finitely many times, reduces to ARMA process [12], [13]. Moreover, Artificial Neural Networks (ANN) that are famous in its application to predict electricity load, recently found its wide range application in price forecasting [14]. In addition, Generalized Autoregressive Conditional Heteroscedastic (GARCH) processes are used to forecast spot market prices [15]. In this paper ARIMA technique is used to predict day-ahead market prices.

Generally, the ARMA(p,q) process can be expressed in the following form: $\phi(B) \lambda_{t}=\theta(B) \varepsilon_{t}$; where $\lambda_{t}$ is the spot market price at time t, $\phi$ and $\theta$ are respectively p-th and q-th degree polynomials

$\phi(\lambda)=1-\phi_{1}-\ldots-\phi_{p} \lambda^{p}$,

$\theta(\varepsilon)=1+\theta_{1}+\ldots+\theta_{q} \varepsilon^{q}$

and $\mathrm{B}$ is backward shift operator defined by $B^{j} \lambda_{t}=\lambda_{t-j}$. In addition, $\varepsilon_{t}$ is white noise sequence, which is independently and identically distributed with 0 mean and $\sigma^{2}$ variance: $\varepsilon_{t} \sim$ $W N\left(0, \sigma^{2}\right)$.

To build a model which best fits to the historical time series usually requires to follow the following steps:

1. Identification,

2. Estimation,

3. Diagnostic checking or validation,

4. Prediction using the constructed model

1. To produce stationarity the underlying time series should be differenced as many times as needed (the inclusion of the factors of the form $(1-B),\left(1-B^{24}\right)$ and $\left(1-B^{168}\right)$, having a hope that the process under study will be reduced to ARMA process. In addition, the logarithmic transformation of the 
original data might be needed to get more homogenous mean and variance. Then, after having the stationary process the second step is to identify the resulting ARMA process: the order of the $\phi(B)$ and $\theta(B)$ polynomials. The existing tools are autocorrelation (ACF) and partial autocorrelation (PACF) functions of the original data. Fig. 1 shows the ACF and Fig. 2 shows PACF of the logarithmic transformed price data from April 20 to June 23, 2011. In successive trials the newly builded model can be refined studying the ACF and PACF of the residuals.

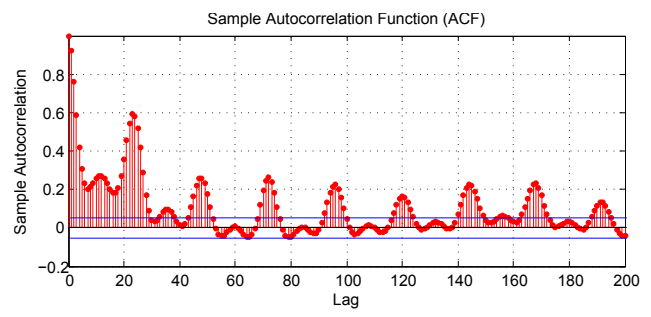

Fig. 1. ACF

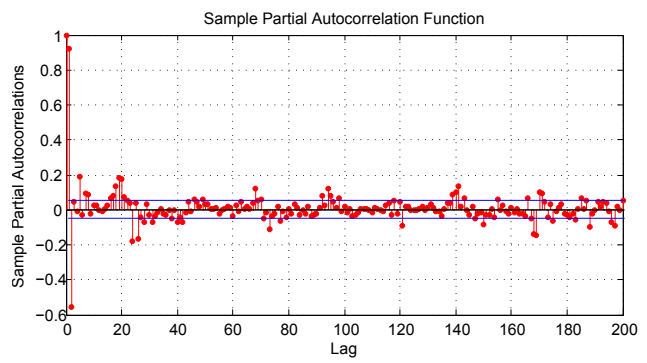

Fig. 2. PACF

2. After completion of the identification process, there is a need to obtain efficient estimates of the parameters in the stochastic model. Maximum Likelihood (ML) method is used for that purpose. Table I displays the estimated parameters.

TABLE I

ESTIMATED PARAMETERS FOR THE SPOT MARKET PRICE MODEL

\begin{tabular}{|l|l|l|l|}
\hline Parameter & Estimate & Parameter & Estimate \\
\hline$\phi_{1}$ & 0.5968 & $\theta_{6}$ & 0.01769 \\
\hline$\phi_{2}$ & -0.418 & $\theta_{7}$ & 0.00627 \\
\hline$\phi_{23}$ & 0.2235 & $\theta_{8}$ & 0.003232 \\
\hline$\phi_{48}$ & 0.03418 & $\theta_{21}$ & 0.015077 \\
\hline$\phi_{72}$ & 0.06269 & $\theta_{47}$ & 0.04521 \\
\hline$\phi_{96}$ & 0.05646 & $\theta_{66}$ & 0.0134 \\
\hline$\phi_{168}$ & 0.08474 & $\theta_{192}$ & -0.0150 \\
\hline$\phi_{192}$ & 0.0085 & $\theta_{215}$ & -0.0042 \\
\hline$\theta_{1}$ & 0.1354 & $\theta_{241}$ & 0.0055 \\
\hline$\theta_{2}$ & 0.0082 & $\theta_{264}$ & -0.0027 \\
\hline$\theta_{3}$ & 0.0503 & $\theta_{288}$ & 0.0083 \\
\hline$\theta_{4}$ & -0.0806 & $\theta_{337}$ & 0.0238 \\
\hline$\theta_{5}$ & 0.0512 & $\theta_{480}$ & -0.0282 \\
\hline & & $\theta_{504}$ & 0.0182 \\
\hline
\end{tabular}

3. The model validation is performed based on the diagnostic cheek, which tests whether the hypothesis about the residuals are true: residuals must be uncorrelated, normally distributed with 0 mean and constant variance. For that purpose ACF and PACF plots of the residuals are studied. Besides, the Ljung-Box test is applied. After the validation step, the model can be used to predict.

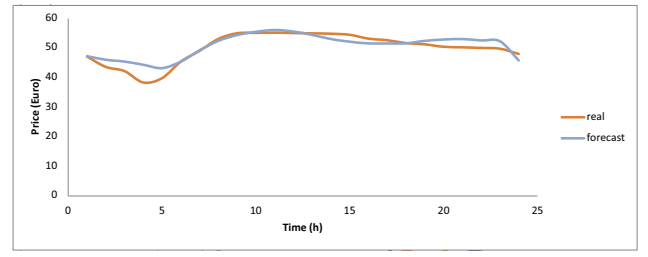

Fig. 3. Real and forecasted prices

4. In this step, the builded model is ready to forecast the next day spot market prices. The constructed final model to predict spot market prices in the Swedish electricity market is the following:

$\left(1-\phi_{1} B^{1}-\phi_{2} B^{2}\right)\left(1-\phi_{23} B^{23}-\phi_{48} B^{48}-\phi_{72} B^{72}-\phi_{96} B^{96}-\right.$ $\left.\phi_{168} B^{168}-\phi_{192} B^{192}\right) \log \lambda_{t}=\left(1-\theta_{1} B^{1}-\theta_{2} B^{2}-\theta_{3} B^{3}-\theta_{4} B^{4}-\right.$ $\left.\theta_{5} B^{5}-\theta_{6} B^{6}-\theta_{7} B^{7}-\theta_{8} B^{8}\right)\left(1-\theta_{21} B^{21}-\theta_{47} B^{47}-\theta_{66} B^{66}-\right.$ $\theta_{192} B^{192}-\theta_{215} B^{215}-\theta_{241} B^{241}-\theta_{264} B^{264}-\theta_{288} B^{288}-$ $\left.\theta_{337} B^{337}-\theta_{480} B^{480}-\theta_{504} B^{504}\right) \varepsilon_{t}$

Fig. 3 demonstrates the forecasting results.

All those steps are implemented in MATLAB using GARCH package.

\section{CASE STUdy}

The case study is structured in the following way: first background information and general comments on the results are stated, then sensitivity analysis in terms of the water opportunity cost is made, and finally profound sensitivity analysis in terms of VSS (the value of the stochastic solution) is provided for the initial case, general case and small reservoir case.

\section{A. Background}

For the case study three cascaded reservoirs are studied. The upper reservoir is larger, which is followed by two smaller reservoirs. Every reservoir has an inflow stream. In addition, each reservoir has a power station which contains a turbine, which by transferring the potential energy of the water to electrical energy generates electricity. Afterwards, the water flows downstream and is stored in the lower plant's reservoir, until released through the lower plants turbine.

The data for the initial reservoirs content and the inflow level are according to Swedish hydropower plants. Inflow data corresponds to week 16 in 2008 . For the spot market price prediction, the data is taken from Nord Pool webcite from april 20 to june 23, 2010. Table II summarizes the maximum storage capacity, the maximum design flow, the maximum production capacity for each power plants and water delay time.

As it was stated above, the random variables in the stochastic problem have continuous distribution. To make the problem solvable, the continuous distribution is discretised and approximated by a scenario tree. This scenario tree should be 
TABLE II

DATA FOR POWER PLANTS

\begin{tabular}{|l|l|l|l|l|}
\hline & $\bar{m}_{j}(\mathrm{HE})$ & $\bar{Q}_{j}\left(m^{3} / s\right)$ & $\bar{G}_{j}(\mathrm{MW})$ & $\tau_{j}(\mathrm{~h})$ \\
\hline I & 305856 & 340 & 95 & 0 \\
\hline II & 1392 & 310 & 50 & 0.5 \\
\hline III & 4008 & 330 & 90 & 2 \\
\hline
\end{tabular}

assessed based on the decision it generates: how close is the approximated solution to the 'true' solution. For that reason the stability requirement has to be fulfilled. For this problem in-sample stability is tested: first, several trees with different number of scenarios are generated, then the optimization problem is solved for each tree and finally solutions are compared [16]. Based on the simulation results, ten scenarios seem sufficient to get stable results. Thus, ten scenarios are generated for the day-ahead market prices and ten scenarios for the water inflow level to each reservoir respectively.

The two-stage stochastic programming problem is formulated in GAMS. CPLEX 12.2 solver is used to solve the resulting mixed integer linear programming model and the execution time is 4.48 seconds. Based on the solution the bidding curve for each hour has been drawn. Fig. 4 and Fig. 5 demonstrates the results. The bidding curve in the Fig. 4 corresponds to the first, fifth and sixth hours and the curve in the Fig. 5 corresponds to the remaining hours. According to the bidding curves; bidding volume is less than total maximum capacity of the three power plants only in the first, fifth and sixth hours. In the remaining hours it bids with the maximum capacity (235 MWh).

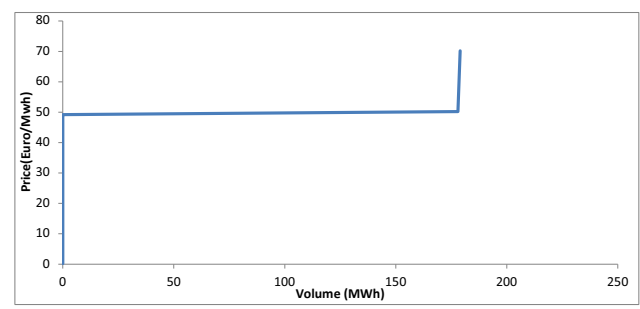

Fig. 4. Bidding curve for the first, fifth and sixth hours

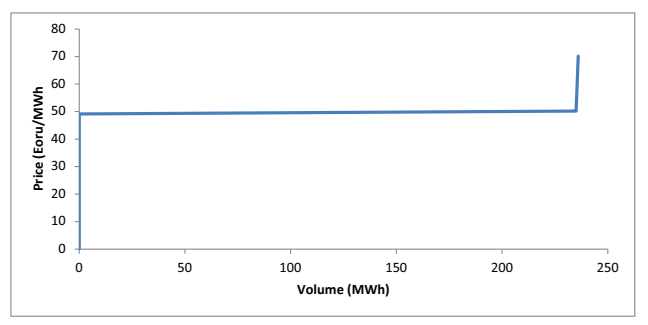

Fig. 5. Bidding curve for the remaining hours

The solution (Fig. 4 and Fig. 5) shows that with this condition there is no bottleneck. A bottleneck may be defined as the part of the system which either causes a forced generation of the electricity while the prices are not so attractive or causes water spillage. According to the solution, for each hour it only bids when the expected market prices are higher than water opportunity cost: $50 \mathrm{Euro} / \mathrm{MWh}$. The possible explanation is nearly the same maximum discharge level in all power plants. Having huge difference in the maximum reservoir capacity, first and second power plants have nearly the same maximum discharge levels. Thus, the water inflow is nearly equal to the water outflow. However, the bottleneck occurs in the system when the initial level of reservoirs is considered more than 80 percent of its maximum reservoirs respectively. In this case the model provides more flexible solution, it starts to bid at 35 Euro/MWh. Apparently, there is a forced release of water under the low market prices to avoid later spillage.

\section{B. Sensitivity analysis in terms of the water opportunity cost}

The sensitivity of the stochastic model is tested in terms of water opportunity cost. When it is taken 55 Euro/MWh instead of 50 Euro/MWh, it only bids for a few hours. When the water opportunity cost is 60 Euro/MWh, it only bids for one hour. As expected, the solution is very sensitive to the changes in the water future value. Thus, it is logical to conclude that, the introduction of the uncertainty related to the water opportunity cost might have a strong effect on the solution.

\section{Sensitivity analysis in terms of VSS: Initial case}

For the comparison purposes and further analysis also the deterministic version of the model is constructed. In the deterministic problem random variables (prices and inflows) are replaced by their expected values respectively. This is called the expected value (EV) problem or mean value problem [9]. Then, the expected value (EEV) is calculated using the EV solution.

In order to assess the value of knowing and using distributions on future outcomes, the VSS concept is used in stochastic optimization [9]. Therefore, to assess the possible gain from the stochastic model: the effect of including the uncertainties in the model, the VSS quantity is calculated. The VSS is simply the difference of the optimal value of the stochastic problem and the EEV results. Table III demonstrates the calculation result (first row).

TABLE III

CALCULATION RESULTS FOR DIFFERENT CASES

\begin{tabular}{|l|l|l|l|}
\hline & SP & EEV & VSS \\
\hline Base case & 4226871 & 4223197 & 3674 \\
\hline High price vol. & 4243480 & 4222098 & 21382 \\
\hline Low price vol. & 4222352 & 4222301 & 51 \\
\hline High inflow vol. & 4226709 & 4223040 & 3669 \\
\hline Low inflow vol. & 4226610 & 4222933 & 3677 \\
\hline Higher inflow & 4340100 & 4336491 & 3609 \\
\hline Lower inflow & 4169313 & 4165625 & 3688 \\
\hline
\end{tabular}

According to the figures in the Table III, VSS $=3674$ Euro for the base case. This is the gain we get using the stochastic model rather than the expected value problem for only one day. Thus, the motivation using the stochastic approach will be stronger if we consider yearly profit.

Moreover, to check the sensitivity of the stochastic model towards price volatility and water inflow level volatility, the 
model is run with the high/low volatile price scenarios and high/low volatile inflow scenarios: for the high volatile cases the standard deviation is taken double compared with the base case and for the low volatile cases standard deviation is taken half of the base case. Then VSS values are calculated for each case and are shown in Table III. The comparison in the VSS values shows that noticeably bigger changes happen when different price volatility levels are considered. Small changes in VSS values occur, when the models are run with high volatile water inflow levels and comparatively low volatile water inflow levels Fig. 6 . These results motivate to investigate the effect of considering water inflow level uncertainty further. For that purpose the cases are considered for which the mean of the water inflow level is varied: first the mean is taken double as big as in the base case, and then the mean is taken half of the mean in the base case. Again the results demonstrate slightly changes in the VSS values (last two rows in Table III and last two bars in Fig. 6).

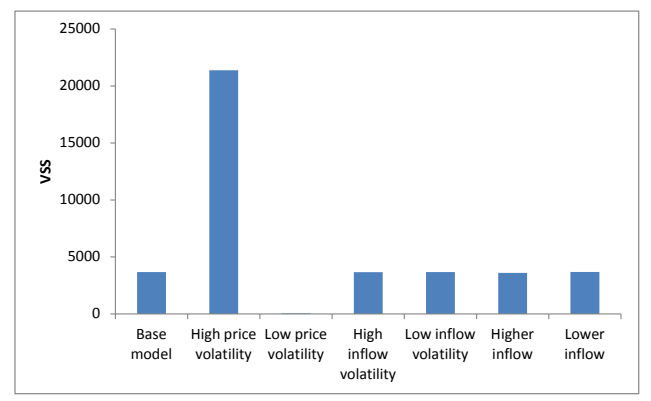

Fig. 6. VSS results for different cases

This comparison results with the VSS values summarized in table 4 tends to indicate that the effect including price uncertainty in the model is higher compered with the effect of considering water inflow level uncertainty.

\section{Sensitivity analysis in terms of VSS: general case}

To generalize the findings, all these scenarios are studied for a case where three cascaded reservoirs with nearly identical maximum storage capacity is considered. The calculated results are summarized in Table IV and also drawn in Fig. 7. Again no observable changes in VSS values occur in terms of the cases considered for inflow level. Thus, the conclusion stated in the previous section is not altered.

TABLE IV

CALCULATION RESULTS: GeNERAL CASE

\begin{tabular}{|l|l|l|l|}
\hline & SP & EEV & VSS \\
\hline Base case & 2874921 & 2871231 & 3690 \\
\hline High price vol. & 2891528 & 2870132 & 21450 \\
\hline Low price vol. & 2870386 & 2870335 & 51 \\
\hline High inflow vol. & 2874769 & 2871074 & 3695 \\
\hline Low inflow vol. & 2874667 & 2870967 & 3700 \\
\hline Higher inflow & 2988203 & 2984513 & 3690 \\
\hline Lower inflow & 2817368 & 2813658 & 3710 \\
\hline
\end{tabular}

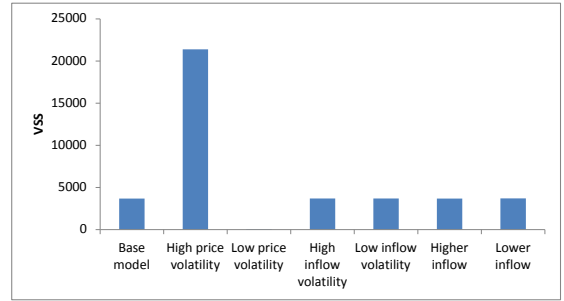

Fig. 7. VSS results for different cases: General case

\section{E. Sensitivity analysis in terms of VSS: small reservoir case}

In order to create a situation, where there is an impact of inflow level uncertainty in the model, all those cases are considered, where three cascaded small reservoirs is studied. The results are demonstrated in the Table V and Fig. 8. From both Table V and Fig. 8 it is possible to see that now there is noticeable change in VSS values for the cases of higher inflow and lower inflow (where mean inflow level is taken double/half as big as in the base case respectively). However, even in this specific structure, which was designed on purpose to see the impact of the inflow level uncertainty, changes in VSS values for inflow level for mentioned above cases are less significant compared with the changes in VSS values in high/low price volatility cases. All these scenarios tend to emphasize the previously drawn conclusion: the impact of including the price uncertainty in the model is higher than that of inflow level uncertainty. This might be because of physical characteristics of the inflow: in contrast with the day-ahead market prices within short time period the changes in the inflow level cannot be sharp to have a strong effect. Furthermore, VSS values for all cases are higher for the small reservoir system as seen in Table III,IV,V.

TABLE V

CALCUlation Results: SMALl RESERVOIR CASE

\begin{tabular}{|l|l|l|l|}
\hline & SP & EEV & VSS \\
\hline Base case & 217274 & 209293 & 7981 \\
\hline High price vol. & 233997 & 206108 & 27889 \\
\hline Low price vol. & 213502 & 213358 & 144 \\
\hline High inflow vol. & 216829 & 208821 & 8008 \\
\hline Low inflow vol. & 217032 & 208975 & 8057 \\
\hline Higher inflow & 326230 & 306788 & 19442 \\
\hline Lower inflow & 159207 & 155201 & 4006 \\
\hline
\end{tabular}

\section{CONCLUSION}

The ongoing growth in wind power increases the uncertainty in the power production. Thus, there is a need for the improved planning tools which will consider those stochasticities. The model presented in this paper is an optimal bidding strategy to the day-ahead market. Two-stage stochastic programming is used to deal with uncertainties related to the day-ahead market 


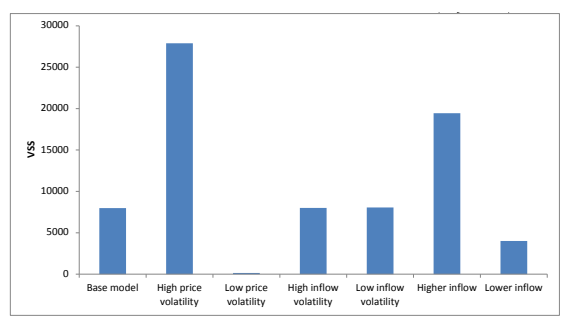

Fig. 8. VSS results for different cases: General case

prices and water inflow levels. The solution clarifies what is profitable for the price-taker hydropower producer; to sell the electric power to the spot market or to store the water for the future production.

A case is studied based on the Swedish hydropower plants, where ten representative scenarios are generated for the prices and inflow levels. The comparison of the optimal value of the stochastic program with the expected result of the expected value problem shows the consequence of considering uncertainties in the problem. Moreover, deep sensitivity analysis is provided which shows that for the short-term hydropower planning problems the effect of considering price uncertainty in the stochastic model is higher compared with considering inflow level uncertainty.

The model further can be improved by considering the joint probability distribution of the unknown variables, if test shows that there is a strong correlation. Moreover, the probability distribution of the real-time market prices and water future values can be included in the model.

\section{REFERENCES}

[1] L. E, "Strategies and decision support systems for integrating variable energy resources in control centers for reliable grid operations," tech. rep., 2011.

[2] S.-E. Fleten and T. K. Kristoffersen, "Stochastic programming for optimizing bidding strategies of a Nordic hydropower producer," European Journal of Operation Research, vol. 181, pp. 916-928, 2007.

[3] S.-E. Fleten and T. K. Kristoffersen, "Short-term hydropower production planning by stochastic programming," Computers and Operations Research, vol. 35, pp. 2656-2671, 2008.

[4] A. J. Conejo, F. J. Nogales, and J. M. Arroyo, "Price-taker bidding strategy under price uncertainty," in Proc. IEEE Power Engineering Society General Meeting, vol. 2, 2003.

[5] M. Olsson and L. Söder, "Hydropower planning including trade-off between energy and reserve markets," in Proc. IEEE Bologna Power Tech, vol. 1, 2003.

[6] A. B. Philpott and H. Craddock, M. Waterer, "Theory and methodology: hydro-electric unit commitment subject to uncertain demand," European Jornals of Operation Research, vol. 125, pp. 410-424, 2000.

[7] J. G. Gonzalez, R. M. R. Muela, L. M. Santos, and A. M. Gonzalez, "Stochastic joint optimization of wind generation and pumped-storage units in an electricity market," IEEE Transactions on Power Systems, vol. $23,2008$.

[8] P. Kall and S. W. Wallace, Stochastic Programming. John Wiley and Sons Inc., 1995.

[9] J. R. Birge and L. F., Introduction to stochastic programming. SpringerVerlag New York Inc., 1997.
[10] N. Nilsson, L. Söder, and D. Sjelvgren, "Integer modelling of spinning reserve requirements in short term scheduling of hydro systems," IEEE Transactions on Power Systems, vol. 13, 1998.

[11] J. Contreras, R. Espinola, F. J. Nogales, and A. J. Conejo, "Arima models to predict next-day electricity prices," vol. 18, no. 3, pp. 1014-1020, 2003.

[12] G. E. P. Box and G. M. Jenkins, Time Series Analysis forecasting and control. HOLDEN-DAY, 1976.

[13] P. J. Brockwell and R. A. Davis, Time Series: Theory and Methods. Springer-Verlag New York Inc., 1991.

[14] B. Szkuta, L. Sanabria, and T. Dillon, "Electricity price short-term forecasting using artificial neural networks," 1999.

[15] R. C. Garcia, J. Contreras, M. van Akkeren, and J. B. C. Garcia, "A garch forecasting model to predict day-ahead electricity prices," vol. 20 , no. 2, pp. 867-874, 2005.

[16] M. Kaut, H. Vladimirou, S. W. Wallace, and S. A. Zenios, "Stability analysis of a portfolio management model based on the conditional value-at-risk measure," 2003. 